\title{
HEALTHCARE SUPERVISING SYSTEM TO MONITOR HEART RATE TO DIAGONIZE AND ALERT HEART ATTACK USING IOT
}

\author{
Dr.E.Punarselvam ${ }^{1,}$ N.Gokul ${ }^{2}$, J.Jeyakumar ${ }^{2}$, R.Lenin ${ }^{2}$, S.Praveenkumar ${ }^{2}$ \\ E-Mail:punarselvam83@gmail.com,hod.it@mec.edu.in \\ 1 Head of the Department, 2. Final Year Student \\ Department of Information Technology, Muthayammal Engineering College, Tamilnadu,
}

\begin{abstract}
These days we have an increased number of heart diseases including increased risk of heart attacks. Our proposed system users sensors that allow to detect heart rate of a person using heartbeat sensing even if the person is at home. The sensor is then interfaced to a microcontroller that allows checking heart rate readings and transmitting them over internet. The user may set the high as well as low levels of heart beat limit. After setting these limits, the system starts monitoring and as soon as patient heart beat goes above a certain limit, the system sends an alert to the controller which then transmits this over the internet and alerts the doctors as well as concerned users. Also the system alerts for lower heartbeats. Whenever the user logs on for monitoring, the system also displays the live heart rate of the patient. Thus concerned ones may monitor heart rate as well get an alert of heart attack to the patient immediately from anywhere and the person can be saved on time.This value will continue to grow if no proper solution is found. Internet of Things (IoT) technology developments allows humans to control a variety of high-tech equipment in our daily lives. One of these is the ease of checking health using gadgets, either a phone, tablet or laptop. we mainly focused on the safety measures for both driver and vehicle by using three types of sensors: Heartbeat sensor, Traffic light sensor and Level sensor. Heartbeat sensor is used to monitor heartbeat rate of the driver constantly and prevents from the accidents by controlling through IOT.
\end{abstract}

\section{KEYWORD: Heart Beat Sensor, IOT, Heart Level.}




\section{INTRODUCTION}

Internet of Thing (IOT) has a major growth in this changing world to innovate new ideas to make things smarter. As per the recent survey published by World Health Organization (WHO) reveals that most of the accidents in India occur due to the cardiac arrest while driving. In the below figure1.1, PIC Micro Controller is used to implement things easier in IOT. Heartbeat sensor is used, which monitors driver's heartbeat rate before he takes off. Traffic Light sensor is also used to avoid accidents by following traffic rules and regulations. Fuel Level sensor helps driver to locate a Petrol stations nearby if the fuel is not sufficient to reach the destination.

IoT relies on a wide range of materials, network infrastructure, communication protocols, Internet services, and computing technologies. Among the range of different technologies involve in the IoT concept, WSN is one of the most important technologies that enable the integration of sensing devices into IoT ecosystems.

Sensing devices are deployed in network to seamlessly collect and send in real-time raw data through the Internet to reach a data center. End users can remotely control the devices using Internet services. They can also access the data center via Internet anytime from anyplace in order to retrieve, process, and analyze data. IoT architecture is an open architecture based on multi-layers. Services-oriented architecture is one of the approaches that have been adopted by researchers in recent years to implement IoT system.

The layers interact with each other by offering different services such as sensing, transmission, collection, storage, and information processing. IoT devices and sensors suffer from computational and energy constraints. Therefore, to achieve interoperability across the heterogeneous networks and seamlessly allow data exchange throughout IoT system, different protocols, and standards are established.

This concept is associated with the Internet of Things (IoT), in which objects sense, and use IPs to communicate among themselves and share information about their environment anytime from anyplace. Wireless Sensors Network (WSN) is considered as one of key technologies of IoT and it is widely used in various areas such as healthcare systems, environmental monitoring systems, Structural Health Monitoring (SHM) systems, etc. 
As novel idea, IoT has rapidly become an attractive topic for researchers and industries. Its integration into monitoring systems like SHM will be advantageous to Industries, businesses, consumers, environment, individuals, and society. The idea behind SHM is to collect data from multiple sensors installed on structures in order to process and extract useful information about current state of the structure for maintenance and safety purpose.

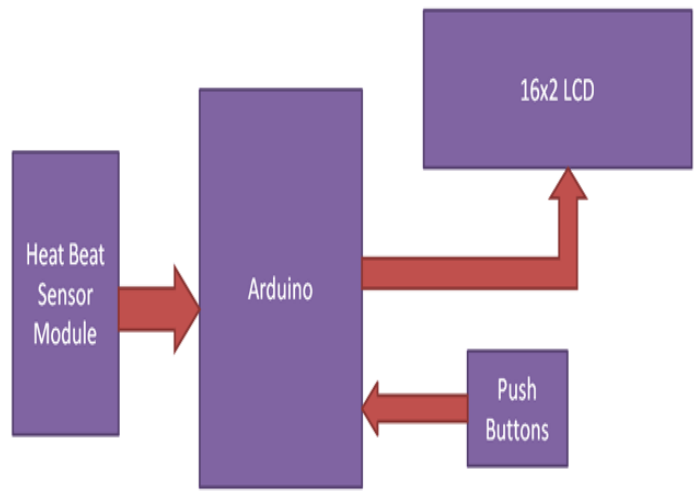

Figure 1Architecture Diagram

\section{IMPLEMENTATION}

The Arduino Uno Arduino, it is a microcontroller board. It is based on ATmega328. Moreover, there are 14 digital input and output pins of which six can be usage as PWM outputs. RX and TX pins are utilize for communication between arduinoboard, computer or additional devices for serial communication. It has operating voltage of 5V.The ATmega 328 has $32 \mathrm{~KB}$ of flash memory for storing code. The ICSP(in-circuit serial programming) header will permit us to use an outside programmer to upload software to our microcontroller unit.

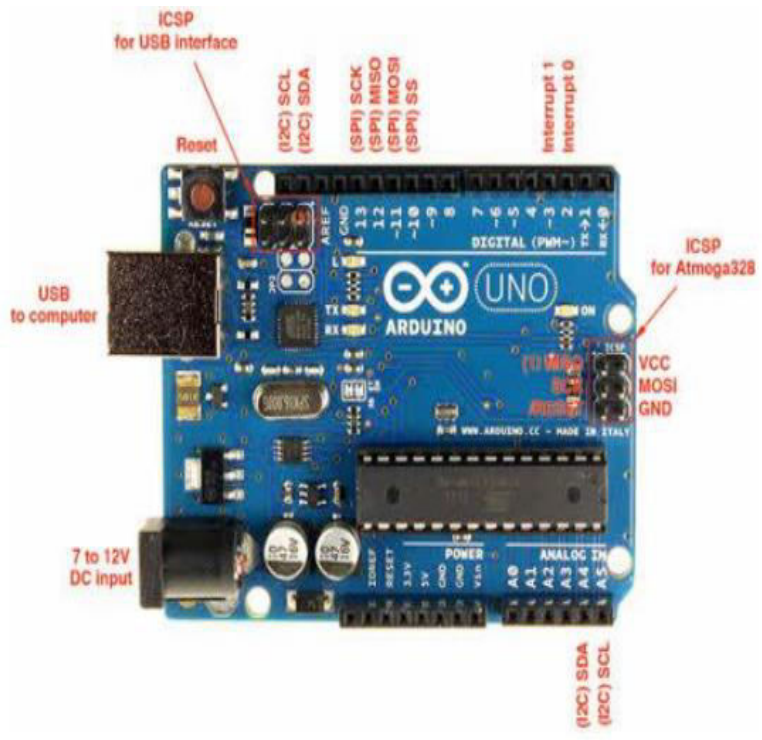

Figure 2 Arduino Microcontroller

\section{ESP8266 Arduino Core}

As Arduino.cc began developing new MCU boards based on non-AVR processors like the ARM/SAM MCU and used in the Arduino Due, they needed to modify the Arduino IDE so that it would be relatively easy to change the IDE to support alternate tool chains to allow Arduino $\mathrm{C} / \mathrm{C}++$ to be compiled down to these new processors. They did this with the 
introduction of the Board Manager and the SAM Core. A "core" is the collection of software components required by the Board Manager and the Arduino IDE to compile an Arduino $\mathrm{C} / \mathrm{C}++$ source file down to the target MCU's machine language. Some creative ESP8266 enthusiasts have developed an Arduino core for the ESP8266 WiFiSoC that is available at the GitHub ESP8266 Core webpage. This is what is popularly called the "ESP8266 Core for the Arduino IDE" and it has become one of the leading software development platforms for the various ESP8266 based modules and development boards, including NodeMCUs. For more information on all things ESP8266, check out the ESP8266 Community Forum on GitHub.

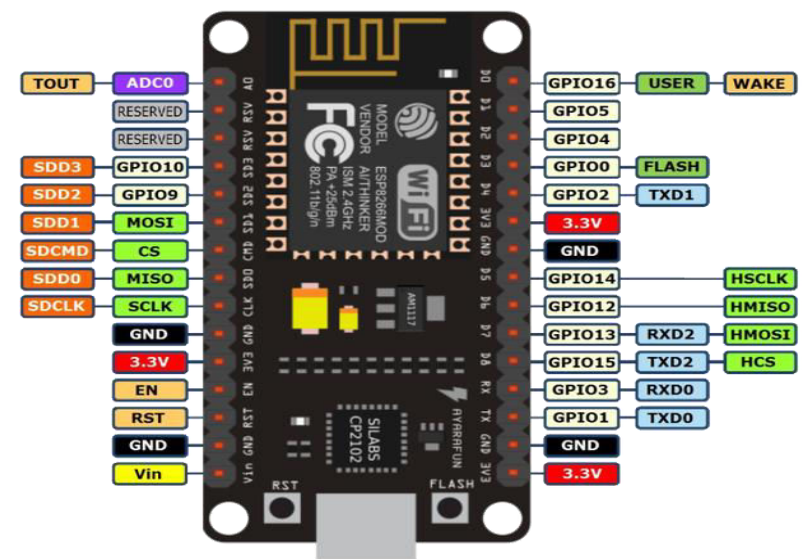

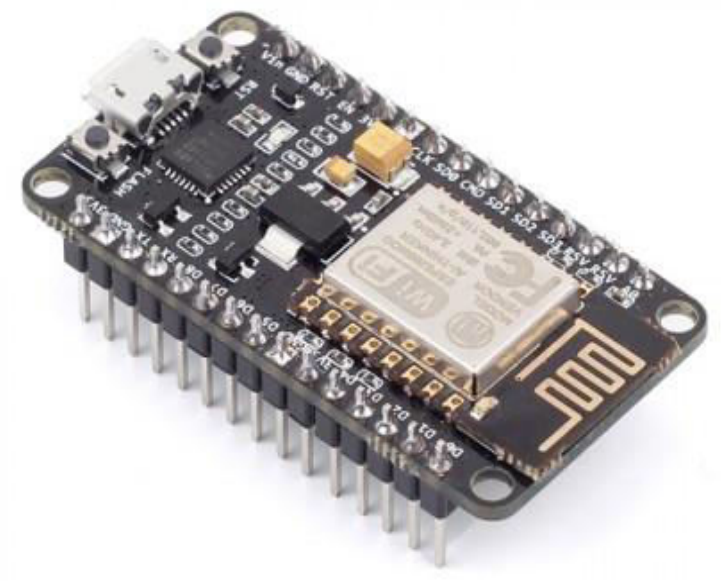

Figure 3 NodeMCU ESP8266 WiFi

\section{Module}

\section{EXISTING SYSTEM}

Now a day there is no automatic medication surveillance based health care system. Everything is done and monitored manually using man power. Heavy machines are used to monitor the patient details in hospitals, which requires huge space and power supplies while processing. Efficiency is so poor compare to the latest technologies such as Internet of Things (IOT).

\subsection{DISADVANTAGES}

- There is no proper intimation system.

- Big Devices are required to monitor the patient and report the details.

- Limited hospital resources, connectivity and range is available, so care takers are restricted to enquire the patient details only with 
doctor and they have to believe them.

- High cost and more time consumption

\section{PROPOSED SYSTEM}

To monitor heartbeat rate of driver heartbeat sensor will be attached in Seat belt of the car. Once the driver starts the vehicle and wears the seat belt, this sensor automatically monitors the heartbeat rate of the driver through Internet of Things. When the heartbeat rate goes abnormal (i.e., above the reference value), IOT notifies the emergency message to the owner of the car, nearby Ambulance and the Police to prevent from accident and save the driver's life. This emergency message will be conveyed to the respective person via GSM. Main concept is if and only if the seat belt is buckled by driver then only engine is allowed to start this makes drivers to buckle up the seat belts compulsory to drive car. This is done by small concept that is the message will be send from heartbeat sensor which is fixed in seatbelt then only the PIC microcontroller allow or permit the driver to start engine.

\subsection{ADVANTAGES}

- Simplicity, mobility and low price.
- This system has the ability of using by common people, especially children and aged people, and don't need any special training.

- Power consumption is low.

- Compatible in size.

\section{RESULTS AND DISCUSSION}

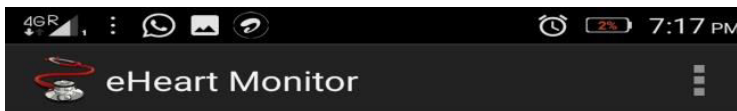

IOT Based Heart Beat Monitoring

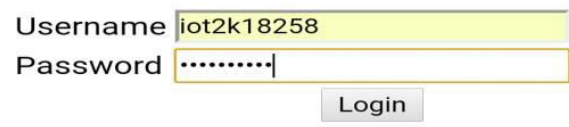

Figure 4. User name and password verification for IOT based heart beat monitoring system

\section{HOME PAGE}

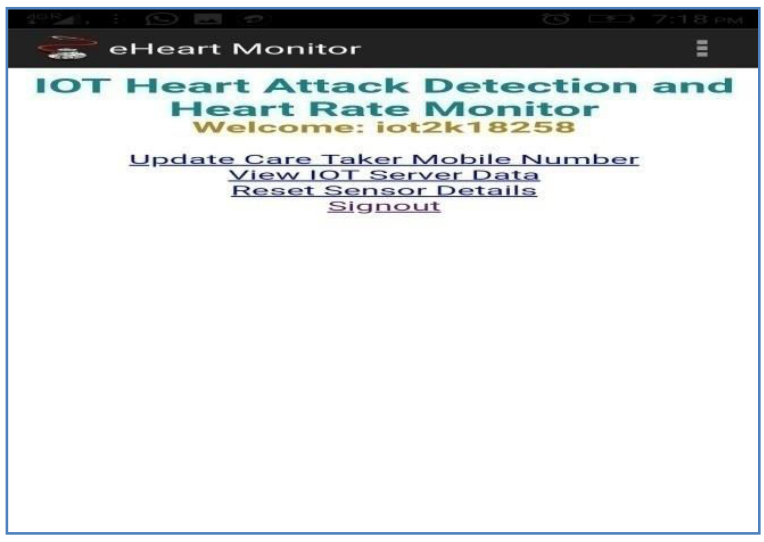

Figure 5 Home Page 


\section{IOT BOARD}

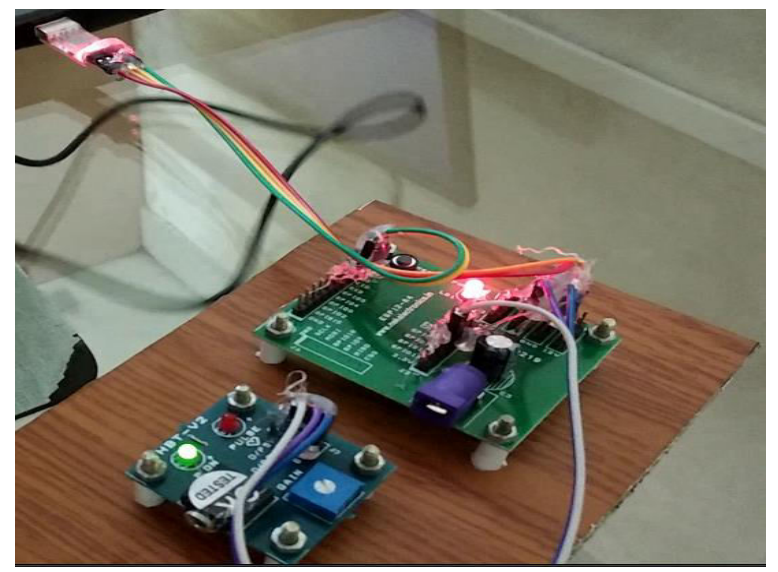

Figure 6 IOT Board

\section{MESSAGE NOTIFICATION}

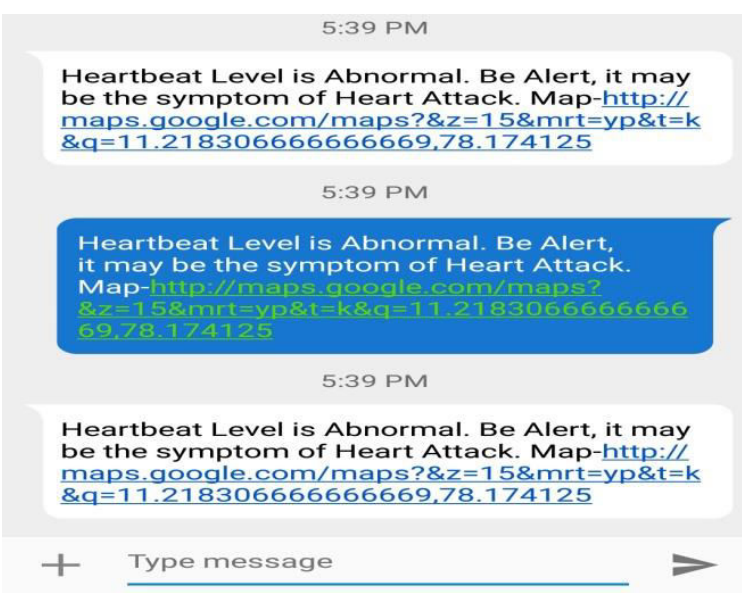

Figure 8 Message Notification

\section{CONCLUSION}

HEART BEAT NORMAL STAGE

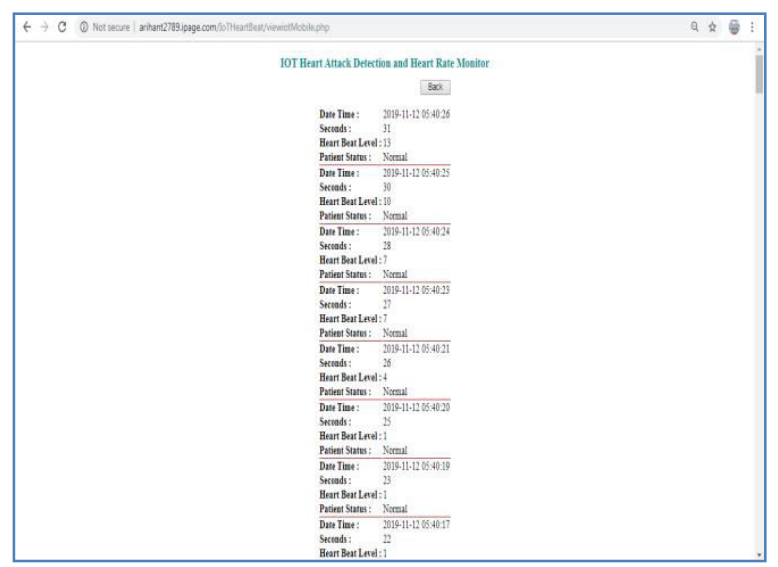

Figure 7 Hearts Beat Normal Stage
We have proposed the smart car system using IOT, GSM and sensors like heartbeat sensor, Fuel level sensor, traffic light sensor and alcohol sensor for prevent the persons and driver travelling in the car from accident. We used "Prevention is better than cure" proverb which makes our life safe and secured. Like the proverb in this paper we described our concept which makes the drivers to follow the road rules to drive the car. Our Government asked drivers to follow the Traffic rules, but drivers refused to follow road rules like buckle up seatbelt, don't drink and drive vehicle, stop in red signal. If we implement the concept described in this modern world then surely there will be reduction in accidents. If our concept is implemented in this real world, 
then the some more implementation is needed to make this project completely useful to this world. We have to fix RFID tag for ambulances, fire engines, etc.., to make signals to detect there is some emergency, so if the RFID tag is detected by traffic signal then the signal will be change from the red to green. If the RFID tag is goes out of range then the signal will change again from green to red.

\section{FUTURE ENHANCEMENTS}

It can be seen that the choice of wireless technologies, which address Structural Health Monitoring system deployment based on IoT, is extremely large. As IoT is a technology that is in trend, a myriad of technologies is being developed to meet all the requirements from IoT community. A number of solutions for IoT communications of Structural Health Monitoring have been proposed in recent years to connect net device, which is able to sense and collect useful information.

\section{REFERENCES}

[1] R. Cohen-Almagor, "Internet history," Int. J. Technoeth., vol. 2, no. 2, pp. 45-64, Apr./Jun. 2011.

[2] P. Suresh, J. V. Daniel, V. Parthasarathy, and R. H. Aswathy, "A state of the art review on the Internet of Things (IoT) history, technology and fields of deployment," in Proc. IEEE Int. Conf. Sci. Eng. Manag. (ICSEMR), Chennai, India, 2014, pp. 1-8.

[3] E.Punarselvam, "Robust Facial Expression Recognition using Local Directional Number Version", International Journal of Innovative Research in Science, Engineering and Technology, ISSN(Online) : 2319 - 8753,ISSN (Print) : 23476710 Vol. 4, Special Issue 6,May 2015,pp 182-186

[4] L. D. Xu, W. He, and S. Li, "Internet of Things in industries: A survey," IEEE Trans. Ind. Informat., vol. 10, no. 4, pp. 2233-2243, Nov. 2014.

[5] Dr.E.Punarselvam, "Effective and Efficient Traffic Scrutiny in Sweet Server with Data Privacy", International Journal on Applications in Information and Communication Engineering Volume 5 : Issue 2: November 2019, PP 1 - 5

[6] .Abawajy J H, Hassan M M. Federated Internet of Things and Cloud Computing Pervasive Patient Health Monitoring System[J]. IEEE 
Communications Magazine, 2017, 55(1):48-53.

[7] Hemant G, Subhas M, Xiang G, et al. WSN- and IoT-Based Smart Homes and Their Extension to Smart Buildings $[\mathrm{J}] . \quad$ Sensors, 2015, 15(5):10350.

[8] Qi J, Yang P, Hanneghan M, et al. Ellipse fitting model for improving the effectiveness of life-logging physical activity measures in an Internet of Things environment $[\mathrm{J}]$. Iet Networks, 2016, 5(5):107-113.

[9] Dr.E.Punarselvam, "Effective and Efficient Traffic Scrutiny in Sweet Server with Data Privacy", International Journal on Applications in Information and Communication Engineering Volume 5: Issue 2: November 2019, PP 1 - 5

[10] Yuenyong S, Nishihara A, Kongprawechnon W, et al. A framework for automatic heart sound analysis without segmentation[J]. Biomedical Engineering Online, 2011, 10(1):13.

[11] Dao A T. Wireless laptop-based phonocardiograph and diagnosis[J]. 2015, 3(3):e1178.
[12] Pérez-Guzmán R E, García-Bermúdez R, Rojas-Ruiz F, et al. Evaluation of Algorithms for Automatic Classification of Heart Sound Signals[C]// International Conference on Bioinformatics and Biomedical Engineering. Springer, Cham, 2017:536-545.

[13] E.Punarselvam, "Big Data using Hadoop Database using python Language to implement Real Time Applications", International Journal of Engineering Research and development,Vol.8 Issue No.12 Oct 2013 PP(19-22) e-ISSN:2278067X,p-ISSN:2278-800X.

[14] Tosi D, Olivero M, Perrone G. Lowcost fibre Bragg grating vibroacoustic sensor for voice and heartbeat detection[J]. Applied Optics, 2008, 47(28):5123.

[15] Dr.E.Punarselvam,"Supervised and Semi Supervised Machine Learning Clustering Algorithm based on feature selection", International Journal on Applications in Information and Communication Engineering, Volume 5 : Issue 2: November 2019, PP 19 -24, ISSN (Online) : $2394-6237$ 